\title{
Analysis of Mode Locking and Ultrashort Laser Pulses With a Nonlinear Refractive Index
}

\author{
JEAN-PIERRE LAUSSADE AND AMNON YARIV, MEMBER, IEEE
}

\begin{abstract}
A new method for locking the longitudinal modes of a laser resonator and generating ultrashort pulses of light is described. The cavity modes are coupled together when a medium with a refractive index nonlinearity is placed in the cavity.

A theoretical study is presented which analyzes the mode structure of a laser resonator containing a cell filled with an anisotropic molecular liquid. It is found that under certain conditions the energy exchange between the modes gives rise to a mode-locked spectrum and to the attendant generation of ultrashort pulses of light $\left(\sim 10^{-11}\right.$ second for a ruby laser, $10^{-12}$ second for a $\mathrm{Nd}^{3+}$ : glass laser).
\end{abstract}

\section{INTRODUCTION}

$\mathrm{T}$ THE OUTPUT electric field of a laser is equal to the sum of the electric fields of the individual modes of the cavity that are amplified by the laser medium, i.e., whose frequencies lie within the gain linewidth $\Delta v_{\alpha}$ of the amplifying transition.

In the normal mode of oscillation of a laser (no perturbation inside the cavity), the phases of the modes are random and uncorrelated, and the output intensity is fluctuating randomly in time around its mean value $N \bar{I}$, where $N$ is the number of oscillating cavity modes and $\bar{I}$ is the average mode intensity.

It has been shown [1]-[3] that if the losses of the laser cavity are modulated at a frequency equal to the intermode spacing frequency $c / 2 L$ ( $L$ is the length of the cavity), mode locking results and the output of the laser consists of a continuous train of pulses that have the following properties.

1) The pulsewidth is equal to the reciprocal of the gain linewidth $1 / \Delta v_{G}$.

2) The pulses are separated in time by the double transit time of the light inside the cavity $2 L / c$.

3) The peak power is equal to $N$ times the average power of the laser where $N$ is the number of coupled modes.

Using internal modulators, ultrashort pulses have been obtained in continuous-wave gas lasers [2] (with a width of $2.5 \times 10^{-9}$ second) and solid-state lasers [3] $\left(8 \times 10^{-11}\right.$ second) with a pulsewidth approaching the theoretical value $1 / \Delta v_{\sigma}$. Internal modulators have also been used to generate ultrashort pulses in pulsed solid-state lasers where

Manuseript received January 6, 1969; revised April 22, 1969 This work was supported by the Army Research Office, Durham, N. C., and the NASA Electronic Research Center.

The authors are with the Division of Engineering and Applied Science, California Institute of Technology, Pasadena, Calif. the duration of the pulsing $(\sim 1 \mu \mathrm{s})$ is larger than the modulating period [4], [5]. The observed pulsewidths were $2 \times 10^{-9}$ second for ruby and $0.5 \times 10^{-9}$ second for Nd:glass, while the theoretical values are, respectively, $10^{-11}$ second and $4 \times 10^{-13}$ second, indicating that the whole linewidth is either not fully mode locked, or that the frequency is swept.

An increase in the output power of solid-state lasers has been obtained by the technique of $Q$ switching [6]. The output of a non-mode-locked Q-switched solid-state laser consists typically of a pulse of $10-50 \times 10^{-9}$ second with a peak power of up to a few hundred megawatts. In these lasers, mode locking has been obtained by inserting a saturable absorber inside the cavity [7], [8]. A saturable absorber is an element whose optical transmission is an increasing function of the intensity of the incident beam. Pulses whose duration is $\sim 10^{-11}$ second as short in ruby lasers and $\sim 10^{-12}$ second in $\mathrm{Nd}$ : glass lasers with peak intensities in excess of $10^{9}$ watts have been observed by using this technique.

In this paper we analyze a new method [9], [10] for generating high-intensity picosecond pulses in $Q$-switched solid-state lasers.

We show theoretically that the introduction of a refractive index nonlinearity inside a laser resonator gives rise to a mode-locked spectrum, characteristic of the ultrashort pulse mode of oscillation. The nonlinearities we consider are provided by liquids consisting of anisotropic uniaxial molecules. These molecules, of which nitrobenzene and $\mathrm{CS}_{2}$ are two representative examples, have different polarizabilities along their axis of symmetry and along any other axis perpendicular to it. We call these polarizabilities $\alpha_{\|}$and $\alpha_{\perp}$ respectively. A linearly polarized electric field applied to such a liquid induces a nonlinear polarization in the medium that is proportional to the difference $\left(\alpha_{\|}-\alpha_{\perp}\right)$ and to the cube of the electric field and therefore produces a change in the dielectric constant of the medium proportional to the square of the electric field. When a liquid with anisotropic molecules is placed inside a laser resonator where the optical electric fields are large enough to produce an appreciable change of the dielectric constant, it couples the longitudinal modes of the resonator together in the following way. Let us assume that three modes of the resonator $(0),(+1)$, and $(-1)$ oscillate with their respective frequencies $\omega_{0}, \omega_{0}+\Omega, \omega_{0}-\Omega . \Omega$ is the radian intermode frequency $\Omega=\pi c / L$. 
The two modes $(0)$ and $(+1)$, for example, induce a change in the dielectric constant of the liquid $\Delta \epsilon \propto E_{0} E_{1}$, where $E_{0}$ and $E_{1}$ are the electric fields of the two modes. Thus $\Delta \epsilon$ has a component oscillating at the frequency $\left(\omega_{0}+\Omega\right)-\omega_{0}=\Omega$. The mode $(-1)$, incident upon the liquid, "sees" a modulation of the dielectric constant at frequency $\Omega$, which causes the generation of sideband frequencies at $\left(\omega_{0}-\Omega\right)+m \Omega, m= \pm 1, \pm 2, \cdots$. As an example, the sideband at $\left(\omega_{0}-\Omega\right)+\Omega=\omega_{0}$ coincides in frequency with mode $(0)$. The effect of the nonlinearity $\Delta \epsilon \propto E^{2}$ is thus seen to be one of coupling modes together, i.e., introducing unique relationships between the amplitudes and the phases of the modes.

We present below a detailed theoretical analysis of the intuitive picture described in the last paragraph. The results of an experimental investigation have been reported elsewhere [9], [10].

The third order nonlinear polarization induced in the laser cavity by the anisotropic molecular liquid is expressed in terms of the parameters of the liquid as a triple summation over the cavity modes. Maxwell's equations with the nonlinear polarization acting as a driving term are then used to find a differential equation obeyed by the modes' amplitudes. A steady-state self-consistent solution is found. This solution applies to practical experimental situations only if sufficient energy exchange takes place between the modes in a time shorter than the duration of a $Q$-switched pulse. The energy exchange time constant $T_{0}$ is calculated in terms of the parameters of the liquid and of a given laser system. We find that under reasonable experimental situations $T_{0}$ can be made short enough so that mode locking can take place during the duration $\sim 10-30 \times 10^{-9}$ second of a typical $Q$-switched laser pulse.

Nonlintear Polarization Induced in the Anisotropic Molecular Liquid

The dipole moment of an anisotropic molecule induced by a linearly $(\| z)$ polarized electric field $E$ along its own direction of polarization is

$$
\mu_{s}=E\left(\alpha_{\|}-\alpha_{\perp}\right) \cos ^{2} \theta+\alpha_{\perp} E
$$

where $\theta$ is the angle between the direction of the electric field and the axis of symmetry of the molecule (see Fig. 1).

The average induced dipole moment of one anisotropic molecule is found by replacing $\cos ^{2} \theta$ by its statistical average $\left\langle\cos ^{2} \theta\right\rangle$ taken over the ensemble of molecules

$$
\left\langle\mu_{2}\right\rangle=E\left(\alpha_{\|}-\alpha_{1}\right)\left\langle\cos ^{2} \theta\right\rangle+\alpha_{\perp} E .
$$

In the absence of any electric field, all the orientations of the molecular axis of symmetry are equally probable and $\left\langle\cos ^{2} \theta\right\rangle=\frac{1}{3}$. In the presence of a strong electric field, the molecules tend to align with their axes parallel to the field direction and $\left\langle\cos ^{2} \theta\right\rangle$ is different from $\frac{1}{3}$. We write

$$
\left\langle\cos ^{2} \theta\right\rangle=s+\frac{1}{3} \text {. }
$$

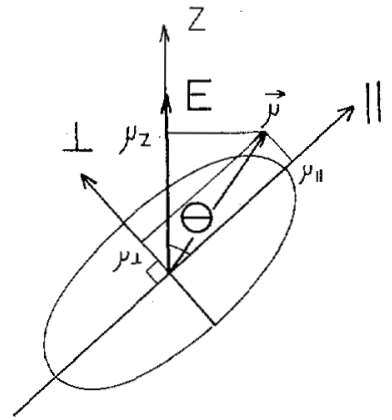

Fig. 1. Orientation of an anisotropic molecule in an electric field.

The quantity $s$ determines the average deviation of the orientation of anisotropic molecules from a purely random orientation. $s$ is the first diagonal element of the anisotropy tensor [11]. From (2) and (3) we obtain

$$
\left\langle\mu_{z}\right\rangle=E\left(\alpha_{\|}-\alpha_{\perp}\right) s+\frac{E}{3}\left(\alpha_{\|}+2 \alpha_{\perp}\right) .
$$

The anisotropy tensor element $s$ can be shown to obey the following differential equation [11]:

$$
\frac{d s}{d t}+\frac{s}{\tau}=\frac{2 \lambda}{3} E^{2}
$$

where $E$ is the linearly polarized electric field. $\tau$ is the time constant with which the molecules regain their random orientation after the electric field has been turned off. It is often called the Debye relaxation time [12] or the orientational relaxation time. $\lambda$ can be shown by a simple thermal equilibrium argument [11] to be given by

$$
\lambda=\frac{1}{15} \frac{\left(\alpha_{\|}-\alpha_{\perp}\right)}{k T \tau} .
$$

We look for a solution of the differential equation (5) in the case where the electric field is the sum of the electric fields of the modes of a laser resonator. For this purpose we find it advantageous to introduce, first, the normal mode formalism for describing the optical resonator field.

\section{Normal Mode Formalism}

In order to describe the mode spectrum of the laser resonator, we introduce a set of orthonormal electric and magnetic vector functions $E_{n}(r)$ and $H_{n}(r)$ as defined by Slater [13], [14]. They are related by the following relationships:

$$
\begin{aligned}
k_{n} E_{n}(r) & =\nabla \times H_{n}(r), k_{n} H_{n}(r)=\nabla \times E_{n}(r) \\
\nabla \cdot E_{n}(r) & =\nabla \cdot H_{n}(r)=0
\end{aligned}
$$

where $k_{n}$ is a constant and $n$ is the index mode number. According to (6) and (7), they satisfy the following differential equations:

$\left(\nabla^{2}+k_{n}^{2}\right) E_{n}(r)=0, \quad\left(\nabla^{2}+k_{n}^{2}\right) H_{n}(r)=0$ 
and they are defined so as to obey the normalization conditions

$$
\int_{V} E_{n}(r) \cdot E_{m}(r) d r=\delta_{n m}, \int_{V} H_{n}(r) \cdot H_{m}(r) d r=\delta_{n m}
$$

The above integrations are performed over the total volume of the cavity.

We express the total electric field $E(r, t)$ (here we go over to a scalar notation appropriate for TEM-like optical resonator modes), and the total magnetic field $H(r, t)$ inside the cavity as

$$
\begin{aligned}
& E(r, t)=-\sum_{n} \frac{1}{\sqrt{\epsilon_{0}}} p_{n}(t) E_{n}(r) \\
& H(r, t)=\sum_{n} \frac{1}{\sqrt{\mu_{0}}} \omega_{n} q_{n}(t) H_{n}(r)
\end{aligned}
$$

where $\omega_{n}$ is defined by

$$
k_{n}=\omega_{n} \sqrt{\mu_{0} \epsilon_{0}}
$$

and where $E_{n}(r)$ and $H_{n}(r)$ are the electric and magnetic scalar functions defined above. The summation is performed over the total number of modes of the cavity. $\epsilon_{0}$ and $\mu_{0}$ are the dielectric constant and the permeability of the medium filling the cavity. $p_{n}(t)$ and $q_{n}(t)$ are unknown functions of time describing the amplitudes and phases of the individual longitudinal cavity modes.

Expression of the Anisotropy Tensor Element $s$ in a Laser Cavity

With the help of the formalism presented above we rewrite the differential equation (5) as follows:

$$
\frac{d s}{d t}+\frac{s}{\tau}=\frac{2 \lambda}{3} \sum_{a} \sum_{b} \frac{1}{\epsilon_{0}} p_{a}(t) p_{b}(t) E_{a}(r) E_{b}(r)
$$

We assume a solution for $p_{n}(t)$ in the form

$$
p_{n}(t)=i \sqrt{\frac{\omega_{n}}{2}}\left(D_{n}^{*}(t) e^{i \omega_{n} t}-D_{n}(t) e^{-i \omega_{n} t}\right)
$$

where $\omega_{n}$ is the optical frequency of the oscillating mode $n$ and $D_{n}(t)$ and $D_{n}^{*}(t)$ are slowly varying functions of time compared to $e^{i \omega_{n} t} . D_{n}^{*}(t)$ is the complex conjugate of $D_{n}(t)$. The phase and amplitude evolution of mode $n$ is thus contained completely in $D_{n}^{*}(t) . p_{a}(t)$ and $p_{b}(t)$ are replaced in (13) by (14). The product $p_{a}(t) p_{b}(t)$ is made up of two frequency components, one at frequency $\omega_{a}+\omega_{b}$ and the other at frequency $\omega_{a}-\omega_{b}$. Both $\omega_{a}$ and $\omega_{b}$ are optical frequencies $\sim 10^{15} \mathrm{rad} / \mathrm{s}$, so $\omega_{a}+\omega_{b}$ is an optical frequency. Since the orientational relaxation time of the liquid is of the order of $10^{-10}$ to $10^{-12}$ second, the molecules cannot respond to fields at optical frequencies. The only term to which the molecules can respond is the term at frequency $\omega_{a}-\omega_{b}$, which ranges from $\omega_{a}-\omega_{b}=0$ to $\omega_{a}-\omega_{b} \sim 2 \pi \Delta \nu_{G}$ where $\Delta \nu_{G}$ is the gain linewidth of the laser medium. Then

$$
\begin{aligned}
& \frac{d s}{d t}+\frac{s}{\tau}= \frac{2 \lambda}{3} \sum_{a} \sum_{b} E_{a}(r) E_{b}(r) \frac{\sqrt{\omega_{a} \omega_{b}}}{2 \epsilon_{0}} \\
& \cdot\left(D_{a}^{*}(t) D_{b}(t) \exp \left[i\left(\omega_{a}-\omega_{b}\right) t\right]+\text { c.c. }\right) .
\end{aligned}
$$

We look for a solution for $s$ in the following form:

$$
s=\sum_{a} \sum_{b} s_{a b}^{*}(t) \exp \left[i\left(\omega_{a}-\omega_{b}\right) t\right]+\text { c.c. }
$$

where $s_{\mathrm{ab}}^{*}(t)$ is a slowly varying function of time compared to $\exp \left[i\left(\omega_{a}-\omega_{b}\right) t\right]$ when $a \neq b$. Therefore for $a \neq b$ we neglect $d s_{a b}^{*}(t) / d t$ with respect to $\left(\omega_{a}-\omega_{b}\right) s_{a b}^{*}(t)$. By substituting (16) for $s$ in (15) we find the following expression for $s_{a b}^{*}(t)$ :

$$
s_{a b}^{*}(t)=\frac{\lambda \tau}{3 \epsilon_{0}} E_{a}(r) E_{b}(r) \sqrt{\omega_{a} \omega_{b}} \frac{D_{a}^{*}(t) D_{b}(t)}{1+i\left(\omega_{a}-\omega_{b}\right) \tau} .
$$

Using this last result in (16) leads to

$$
\begin{aligned}
s=\frac{\lambda \tau}{3 \epsilon_{0}} \sum_{a} \sum_{b} & \sqrt{\omega_{a} \omega_{b}} \frac{D_{a}^{*}(t) D_{b}(t)}{1+i\left(\omega_{a}-\omega_{b}\right) \tau} \\
\cdot & \exp \left[i\left(\omega_{a}-\omega_{b}\right) t\right] E_{a}(r) E_{b}(r)+\text { c.c. }
\end{aligned}
$$

We thus find that the average molecular orientation oscillates at integral multiples of the intermode frequency (since $\omega_{a}-\omega_{b}=m \pi c / L, m= \pm 1, \pm 2, \cdots$ ). The amplitude of each frequency component is proportional to the product of two laser fields $D_{a}^{*} D_{b}$ and lags by $\phi_{a b}=$ $\tan ^{-1}\left[\left(\omega_{a}-\omega_{b}\right) \tau\right]$.

\section{Total Nonlinear Polarization}

The total average polarization $P$ induced in the anisotropic molecular liquid per unit volume is $P=N_{0}\left\langle\mu_{z}\right\rangle$ where $N_{0}$ is the number of molecules per unit volume and $\left\langle\mu_{z}\right\rangle$ is given by (4). In MKS units the displacement vector $D$ is expressed as $D=\epsilon_{0} E+P$, which can be written as $D=\epsilon_{L} E+P_{\mathrm{N} \text {. }}$ where $\epsilon_{L}$ is the dielectric constant of the liquid and $\boldsymbol{P}_{\mathrm{NL}}$ is the total nonlinear polarization induced in the liquid. According to (4) the scalar polarization field is

$$
P_{\mathrm{NL}}=N_{0}\left(\alpha_{\|}-\alpha_{\perp}\right) s E .
$$

$P_{\mathrm{NL}}$ can be expressed in terms of the cavity mode wave functions $D_{n}(t) E_{n}(r)$ with the help of (10), (14), and (18):

$$
\begin{aligned}
P_{N L}= & -3 i \epsilon_{2} \sum_{a} \sum_{b} \sum_{c} \sqrt{\frac{\omega_{a} \omega_{b} \omega_{b}}{8 \epsilon_{0}^{3}}} E_{a}(r) E_{b}(r) E_{c}(r) \\
& \times \frac{D_{a}^{*}(t) D_{b}(t) D_{c}^{*}(t)}{1+i\left(\omega_{a}-\omega_{b}\right) \tau} \exp \left[i\left(\omega_{a}-\omega_{b}+\omega_{c}\right) t\right]+\text { c.c. }
\end{aligned}
$$

where $\epsilon_{2}$ is the optical Kerr constant expressed in MKS units

$$
\epsilon_{2}=\frac{4}{135} N_{0} \frac{\left(\alpha_{\|}-\alpha_{\perp}\right)^{2}}{k T} .
$$




\section{Solution of Maxweli Equations With a \\ Third-Order Nonlinear. Polarization}

In order to find an expression for the electric field in a laser resonator in the presence of an anisotropic molecular liquid, a nonlinear polarization driving term is included in the Maxwell equations:

$$
\begin{aligned}
& \nabla \times E=-\mu_{0} \frac{\partial H}{\partial t} \\
& \nabla \times H=\sigma E+\frac{\partial}{\partial t}\left(\epsilon_{0} E+P_{\mathrm{NL}}\right)
\end{aligned}
$$

where $\sigma$ is the electrical conductivity of the medium. For simplicity, we have assumed that the linear dielectric constant of the liquid is equal to 1 .

By this procedure we first find a differential equation obeyed by the mode amplitudes $D_{n}^{*}(t)$.

\section{Differential Equation for the Mode Amplitude $D_{n}(t)$}

We replace the electric field and magnetic field by their expressions (10) and (11) in the first of the Maxwell equations (22). Both sides of the equation are then multiplied by $H_{m}(r)$ and integrated over the volume of the cavity. With the help of (9) and (12), we find

$$
p_{n}(t)=q_{n}^{\prime}(t) \text {. }
$$

The first Maxwell equation (22) has provided a relationship between $p_{n}(t)$ and $q_{n}(t)$. According to (24) and (14) we can now express $q_{n}(t)$ and then the magnetic field $H(r, t)$ in terms of the complex amplitude $D_{n}^{*}(t)$ of the $n$th cavity mode:

$$
q_{n}(t)=\frac{1}{\sqrt{2 \omega_{n}}}\left(D_{n}^{*}(t) e^{i \omega_{n} t}+D_{n}(t) e^{-i \omega_{n} t}\right)
$$

It has been assumed in deriving expression (25) that $D_{n}(t)$ is a very slowly varying function of time compared to $e^{i \omega_{n} \varepsilon}$, i.e.,

$$
\left|\frac{d D_{n}(t)}{d t}\right| \ll \omega_{n}\left|D_{n}(t)\right|
$$

A second relationship between $p_{n}(t)$ and $q_{n}(t)$ is obtained by using (23) in which $E, H$, and $P_{\mathrm{NL}}$ are replaced by their expressions (10), (11), and (20). Both sides of the equation are multiplied by $E_{m}(r)$ and integrated over the volume of the cavity. The relationships (6) and (9) are used to find the differential equation

$$
\begin{aligned}
\omega_{n}^{2} q_{n}(t)+\frac{\sigma}{\epsilon_{0}} & p_{n}(t)+p_{n}^{\prime}(t) \\
= & \frac{3 \epsilon_{2}}{2 \sqrt{2}} \epsilon_{0}^{2} \sum_{a} \sum_{b} \sum_{c} \sqrt{\omega_{a} \omega_{b} \omega_{c}} S_{n a b c} \\
& \times\left(\omega_{a}-\omega_{b}+\omega_{c}\right)\left(\frac{D_{a}^{*}(t) D_{b}(t) D_{c}^{*}(t)}{1+i\left(\omega_{a}-\omega_{b}\right) \tau}\right. \\
& \left.\cdot \exp \left[i\left(\omega_{a}-\omega_{b}+\omega_{c}\right) t\right]+\text { c.c. }\right)
\end{aligned}
$$

where $S_{n a b c}$ as defined by the relationship

$$
S_{n a b c}=\int_{\substack{\text { volumo of the } \\ \text { ani sotropio tiquid }}} E_{n}(r) E_{a}(r) E_{b}(r) E_{c}(r) d V
$$

is a factor that depends upon the dimensions and the position of the anisotropic liquid inside the laser cavity.

The equation $p_{n}(t)=q_{n}^{\prime}(t)$ has allowed us to express $q_{n}(t)$ in terms of $D_{n}^{*}(t)$ and $D_{n}(t)$. We then replace $p_{n}(t)$ and $q_{n}(t)$ by their expressions (14) and (25) and find a differential equation for $D_{n}^{*}(t)$ instead of $p_{n}(t)$ :

$$
\begin{aligned}
\frac{d D_{n}^{*}(t)}{d t}+\frac{\sigma}{\epsilon_{0}} D_{n}^{*}(t)= & -\frac{3 i \epsilon_{2}}{2 \epsilon_{0}^{2}} \sqrt{\omega_{n}} \sum_{a, b, c} \sqrt{\omega_{a} \omega_{b} \omega_{c}} S_{n a b c} \\
& \cdot \frac{D_{a}^{*}(t) D_{b}(t) D_{c}^{*}(t)}{1+i\left(\omega_{a}-\omega_{b}\right) \tau}
\end{aligned}
$$

where only the modes $a, b$, and $c$ such that

$$
\omega_{a}-\omega_{b}+\omega_{c}=\omega_{n}
$$

can provide synchronous driving of the oscillation at $\omega_{n}$.

The frequency of the $n$th mode is defined as

$$
\omega_{n}=n \Omega
$$

where $\Omega=\pi c / L$ and $n$ is a very large number equal to the number of half-wavelengths contained in the length of the resonator. With the definition (30), the condition (29) becomes $a-b+c=n$. The triple summation over $a, b, c$ in (28) is replaced by a double summation over $m$ and $p$ such that $a=n+m, b=n+m+p$, and $c=n+p$. The above substitution leads to the differential equation

$$
\begin{aligned}
\frac{d D_{n}^{*}}{d t}= & -\frac{3 i \epsilon_{2} \omega_{n}}{2 \epsilon_{0}^{2}} \sum_{p} \frac{D_{n+p}^{*}}{(1-i p \Omega \tau)} \\
& \cdot \sum_{m} \omega_{n+m} D_{n+m \div p} D_{n+m}^{*} S_{n, m, p}
\end{aligned}
$$

where we have assumed that the gain provided by the laser medium is equal to the losses of the cavity (reflection losses at the mirror and diffraction losses), so that the effective conductivity $\sigma$ is taken as zero.

The $n$th cavity mode has the spatial dependence:

$$
E_{n}(r)=\sqrt{\frac{2}{A L}} \sin \frac{n \pi x}{L}
$$

where $A$ is the cross section of the beam. The factor $S_{n, m, p}$ is calculated explicitly, using (27), when a liquid cell of length $2 l$ is inserted in an optical cavity of length $L$ at a distance $L_{0}$ from a mirror that is taken as the origin of the coordinates (see Fig. 2):

$$
\begin{aligned}
S_{n, m, p}= & \frac{1}{V}\left(\frac{l}{L}+\frac{1}{2 p \pi} \sin \frac{2 \pi p l}{L} \cos \frac{2 \pi p L_{0}}{L}\right. \\
& \left.+\frac{1}{2 m \pi} \sin \frac{2 m \pi l}{L} \cos \frac{2 \pi m L_{0}}{L}\right) .
\end{aligned}
$$

Solution of the Differential Equation for $D_{n}^{*}(t)$

We find a solution of the differential equation (31) in the case when the liquid cell fills half of the laser cavity $L_{0}=l=L / 4$. According to (31) and (33), 


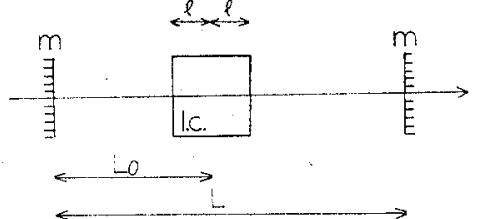

Fig. 2. Position of the liquid cell (lc) inside the cavity formed by the two mirrors $(m)$.

$$
\begin{aligned}
\frac{d D_{n}^{*}}{d t}= & -\frac{3 i \epsilon_{2} \omega_{n}}{2 \epsilon_{0}^{2} V} \frac{l}{L}\left(\sum_{p} \frac{D_{n+p}^{*}}{(1-i p \Omega \tau)} \sum_{m} \omega_{n+m} D_{n+m+p} D_{n+m}^{*}\right. \\
& +D_{n}^{*} \sum_{m} \omega_{n+m} D_{n+m} D_{n+m}^{*} \\
& \left.+D_{n}^{*} \sum_{p} \omega_{n+p} \frac{D_{n+p} D_{n+p}^{*}}{(1-i p \Omega \tau)}\right) .
\end{aligned}
$$

In order to specify the values of $p$ and $m$ over which the summations in (34) are performed, we discuss in some detail the mechanism of power exchange between modes. The above equation describes the coupling between four modes labeled $n, n+p, n+m+p$, and $n+m$. The susceptibility for this coupling is $\chi=\epsilon_{2} /$ $(1-i p \Omega \tau)$. We can write the susceptibility $\chi$ as a real part $\chi^{\prime}$ plus an imaginary part $+i \chi^{\prime \prime}$. The transfer of energy between the modes $n$ and $n+p$ is proportional to $\chi^{\prime \prime}=\epsilon_{2}(p \Omega \tau) /\left[1+(p \Omega \tau)^{2}\right]$.

The rate at which energy is transferred into one mode via another mode separated in frequency by $p \Omega$ is thus proportional to the function $f\left(p / m_{0}\right)=\left(p / m_{0}\right) /\left[1+\left(p / m_{0}\right)^{2}\right]$ where $m_{0}=1 / \Omega \tau$. This function has a maximum for $p=m_{0}$ and decreases to zero for $p>m_{0}$. We neglect the interaction of the $n$th mode with the $n+p$ th mode when $p>2 m_{0}$; the summation over $p$ is then limited to $-2 m_{0}$ and $2 m_{0}$. We label $N_{1}$ and $N_{2}$ the lowest and highest frequency modes of the gain linewidth; the total number of modes is $N=N_{2}-N_{1}$. We look for a solution of (34) in the form

$$
\begin{aligned}
& D_{n}^{*}(t)=\beta(t) e^{i n \Phi(t)} \\
& D_{n}(t)=\beta(t) e^{-i n \Phi(t)}
\end{aligned}
$$

where $\beta(t)$ and $\Phi(t)$ are real functions of time. In this assumed form the cavity modes have the same amplitude $\beta(t)$ and the phase difference between two adjacent modes is the same for all the modes and is equal to $\Phi(t)$. We substitute for $D_{n}^{*}$ and $D_{n}$ in (34) their expressions (35), and perform the summations with the limits described above to find

$$
\begin{aligned}
\left(\frac{d \beta(t)}{d t}+\right. & \left.i n \Phi^{\prime}(t) \beta(t)\right) e^{i n \Phi(t)} \\
= & -\frac{3 i \epsilon_{2} \omega_{n}}{2 \epsilon_{0}^{2} V} \frac{l}{L} e^{i n \Phi(t)} \\
& \times\left(\beta \sum_{p=-2 m_{0}}^{2 m_{0}}\left(\frac{1}{1-i p \Omega \tau}\right) \sum_{m} \omega_{n+m} \beta^{2}+\beta \varepsilon_{T}\right. \\
& \left.+\beta \sum_{p=-2 m_{0}}^{2 m_{0}} \frac{\omega_{m+p} \beta^{2}}{(1-i p \Omega \tau)}\right) .
\end{aligned}
$$

In (36) we have used the fact that the total electromagnetic energy $\varepsilon_{T}$ stored in the cavity can be expressed according to (9)-(11), (14), (25), and (35) as

$$
\begin{aligned}
\mathcal{E}_{T} & =\frac{1}{2} \int_{\substack{\text { volume of } \\
\text { the oavi ty }}}\left(E^{2}+H^{2}\right) d v \\
& =\sum_{m} \omega_{n+m} D_{n+m} D_{n+m}^{*}=\sum_{m} \omega_{n+m} \beta^{2} .
\end{aligned}
$$

The last term in the parentheses on the right-hand side of (36) is roughly equal to $1 / N$ times the sum of the first two terms. Since $N$ is very large, it can be neglected and we write

$\frac{d \beta(t)}{d t}+i n \Phi^{\prime}(t) \beta(t)=-\frac{3 i \epsilon_{2} \omega_{n}}{\epsilon_{0}^{2} V} \frac{l}{L} \varepsilon_{T} \beta \sum_{p=0}^{2 m_{0}}\left(\frac{1}{1+p^{2} \Omega^{2} \tau^{2}}\right)$

The right-hand side of (37) is purely imaginary. Therefore $d \beta(t) / d t=0$ and $\beta(t) \equiv \beta=$ constant. The amplitudes of the cavity modes are constant in time and a solution of (37) is the following:

$$
D_{n}^{*}(t) e^{i \omega_{n} t}=\beta e^{i \omega_{n}}\left(1-\frac{3 \epsilon_{2}}{\epsilon_{0}^{2} V} \frac{l}{L} \varepsilon_{T} \sum_{p=0}^{2 m_{0}}\left(\frac{1}{1+p^{2} \Omega^{2} \tau^{2}}\right)\right) t
$$

Therefore the presence of an anisotropic molecular liquid inside a laser resonator gives rise to a mode-locked spectrum of equal amplitudes and zero phases. The term inside the parenthesis in the exponential represents frequency pulling. It is easy to show that a solution in the form of (38) results even for an arbitrary placement of the cell within the resonator. The time envelope $E(t) \propto$ $\left(\sum_{n} D_{n}^{*}(t) \exp \left(i \omega_{n} t\right)+\right.$ c.c. $)$, where $D_{n}^{*}(t)$ is given by (38), consists of a train of ultrashort laser pulses of very high intensity. These pulses are separated in time by the double transit time of the light inside the cavity $2 L / c$ and approach a duration $T \sim\left(\Delta v_{G}\right)^{-1}$ where $\Delta v_{G}$ is the gain linewidth of the amplifying transition [1]. The resonance frequency of the $n$th mode is slightly pulled from its initial value $\omega_{n}$ by an amount proportional to the stored energy $\varepsilon_{T}$.

\section{Energy Circulation Time Constant}

As a measure of the strength of the mode coupling due to the refractive index nonlinearity, we define a circulation time $T_{0}$ as the exponential time constant for the circulation of the energy in one mode due to its interactions with all others, once phase locking is achieved. If $\varepsilon_{n}$ is the energy of the $n$th mode, then

$$
\frac{1}{T_{0}}=\frac{1}{\varepsilon_{n}} \frac{d \varepsilon_{n}}{d t}
$$

\section{Calculation of $T_{0}$}

The energy of the $n$th mode is $\varepsilon_{n}=\omega_{n} D_{n}^{*} D_{n}=\omega_{n} \beta^{2}$ where $D_{n}^{*}$ and $D_{n}$ have been replaced by their expressions (35). Therefore, according to (39) 


$$
\frac{1}{T_{0}}=2 \frac{\beta^{\prime}}{\beta} .
$$

We have found earlier that $\beta^{\prime}=0$ and $\beta=$ constant. This is true because in the steady state as expressed by (38) and, assuming a very large number of modes, as much energy flows into the $n$th mode via the higher frequency modes as flows out of it via the lower frequency modes. We want to calculate the rate at which energy is flowing into the $n$th mode. Therefore, in order to find $\beta^{\prime}$, we keep in the summation of $p$ of expression (36), only the terms where $p$ is positive.

Only the real part of the right-hand side of (36) gives rise to power exchange between the modes. Equating $\beta^{\prime}(t)$ to the real part of the right-hand side of (36) and using the definition (40) yields

$$
\frac{1}{T_{0}}=\frac{3 \epsilon_{2} \omega_{n}}{\epsilon_{0}^{2}} \frac{l}{L} \frac{\varepsilon_{T}}{V} \sum_{p=1}^{2 m_{0}} \frac{p \Omega \tau}{1+(p \Omega \tau)^{2}} .
$$

The term

$$
\sum_{p=1}^{2 m_{o}} \frac{p \Omega \tau}{1+(p \Omega \tau)^{2}}
$$

accounting for the number of modes interacting with any one mode, is approximately equal to $1 / \Omega \tau$. Therefore

$$
\frac{1}{T_{0}}=\frac{3}{2} \frac{\epsilon_{2} \omega_{n}}{\epsilon_{0}^{2}} \frac{l}{L} \frac{\varepsilon_{T}}{V} \frac{1}{\Omega \tau} .
$$

The rate at which energy is exchanged between modes is proportional to the electromagnetic energy stored per unit volume in the cavity $\varepsilon_{T} / V$, to the ratio of the length of the liquid cell to the length of the cavity $l / L$, to the optical Kerr constant $\epsilon_{2}$, and inversely proportional to $\Omega \tau . \epsilon_{2}$ in (41) is given in MKS units. Using the Kerr constant $B_{0}[15]$ in esu units, $1 / T_{0}$ is expressed in the following way:

$$
\frac{1}{T_{0}}=12 \pi C n \frac{\lambda_{A}}{\lambda} B_{0} \frac{l}{L} \frac{\varepsilon_{T}}{V} \frac{1}{\Omega \tau}
$$

where $\lambda$ is the wavelength of the solid-state laser and $\lambda_{A}$ is the wavelength of the argon laser used in [15] for measuring the optical Kerr constants, $\lambda_{A}=4880 \AA$. For the following experimental situation-a 5 -cm cell containing nitrobenzene $\left(B_{0}=2.9 \times 10^{-7}\right.$ esu, $\tau_{300}{ }^{\circ} \mathrm{K}=$ $5 \times 10^{-11}$ second) and a laser cavity 1 meter long with a beam cross section of $1 \mathrm{~cm}^{2}$ and a total energy of 0.1 joule - we find a circulation time $T_{0}$ of the order of 1 ns and, therefore, sufficient energy exchange between cavity modes is expected to take place to produce efficient mode coupling within the duration of typical $Q$-switched laser pulses $\left(>10^{-8}\right.$ second).

\section{Discussion ANd Conclusion}

The important parameters of the anisotropic molecular liquid are its optical Kerr constant $\epsilon_{2}$ and its orientational relaxation time $\tau$. The rate of energy exchange as ex- pressed by (41) is proportional to $\epsilon_{2}$ and inversely proportional to $\Omega \tau$; therefore, for stronger mode coupling $\Omega \tau$ should be as small as possible. However, there is a lower limit to the possible values of $\Omega \tau$. This is explained by examining the physical significance of this parameter.

The rate at which energy is exchanged between the $n$th mode and the $(n+p)$ th mode, for example, is proportional to the quantity $p \Omega \tau /\left[1+(p \Omega \tau)^{2}\right]$, which is equal to the imaginary part of the nonlinear susceptibility of an anisotropic molecule in an electric field with radian beat frequency $p \Omega$. The maximum of this quantity occurs at $p=1 / \Omega \tau$.

If $\Omega \tau \gg 1$, the molecular orientation does not respond to optical envelope variations at frequency $\Omega$ or higher and the amount of refractive index nonlinearity is too small to couple the modes together.

If $\Omega \tau=1$, the $n$th mode exchanges energy principally with adjacent modes. The rate at which it receives energy from higher frequency modes is maximum for the $(n+1)$ th mode and decreases rapidly for the $(n+2),(n+3)$ modes, and so on. In order to couple more modes faster, the relaxation time $\tau$ of the molecule has to be made shorter so that the molecules respond to more frequency components. But there is a limit to how short $\Omega \tau$ should be. If $\Omega \tau \ll 1 / N$, where $N$ is the total number of oscillating modes, the $n$th mode is coupled most effectively with modes $n+p$ and $n-p$ where $p \gg N$, so that these modes are outside the gain linewidth. In that case most of the energy is coupled outside the gain linewidth and lost without giving rise to any appreciable coupling between the oscillating modes. In Fig. 3 the function $f(p \Omega \tau)=p \Omega \tau / 1+\left(p^{2} \Omega^{2} \tau^{2}\right)$, which is a measure of the mode-coupling strength, is presented for $\Omega \tau=1,1 / N<$ $\Omega \tau<1$ and $\Omega \tau<1 / N$. For producing strong mode coupling, the relaxation time must be chosen so that

$$
\tau \sim \frac{2}{N \Omega}=\frac{1}{\pi \Delta \nu_{\theta}}
$$

and is thus determined by the laser transition. For example, in a ruby laser with a $\Delta \nu_{G} \approx 2 \mathrm{~cm}^{-1}, \tau$ should be $\approx 10^{-11}$ second.

The relaxation time $\tau$ is given by the expression [12] $\tau=\eta V / k T$ where

$$
\begin{aligned}
& \eta=\text { the viscosity of the liquid } \\
& V=\text { the volume of one molecule } \\
& T=\text { the temperature of the liquid. }
\end{aligned}
$$

The viscosity $\eta$ is a decreasing function of temperature [16]. It varies as $A e^{B / T}$ where $A$ and $B$ are two, empirically found, constants characteristic of the liquid. The relaxation time $\tau$ is thus a decreasing function of the temperature. This dependence has been experimentally verified for nitrobenzene by Rank et al. [17] who measured the frequency shift of the stimulated Rayleigh line as a function of temperature from $T=12$ to $117^{\circ} \mathrm{C}$.

The relaxation time of the anisotropic molecular liquid 


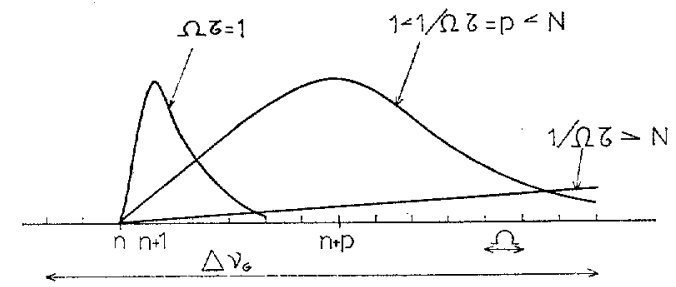

Fig. 3. The strength of the mode coupling, as represented by the function $p \Omega \tau /\left[1+(p \Omega \tau)^{2}\right]$, is a function of $\Omega \tau$.

can be adjusted by controlling its temperature. For the optimum value of $\tau$ given above, nitrobenzene with a room temperature relaxation time of $50 \times 10^{-12}$ second requires heating, while carbon disulfide with a room temperature relaxation time of $10^{-12}$ second requires cooling.

The dependence of mode locking on the proper value of the molecular orientation time $\tau$ and its manipulation by temperature has been verified experimentally [9], [10].

One important problem that we have not considered is the following. We have shown that a nonlinear refractive index can give rise to a steady-state mode locking via physical exchange of energy between the longitudinal modes of the laser. What we have not shown, however, is that starting from a nonlocked mode spectrum (i.e., arbitrary phases) the effect of the nonlinearity is to force the phases to lock. That this may be the case is suggested by the model of pulse steepening in a medium in which the index of refraction increases with the wave intensity [18]. This point is left open and it is hoped that a numerical transient analysis now in progress will help clarify it.

In conclusion, an analysis has been presented that treats the problem of multimode laser oscillation in the presence of a nonlinear dielectric medium. The steadystate laser field corresponds to mode-locked oscillation of the type giving rise to ultrashort pulses.

\section{ACKNOWLEDGMENT}

The authors gratefully acknowledge many fruitful discussions with J. Comly and Dr. E. Garmire.

\section{REFERENCES}

[1] L. E. Hargrove, R. L. Fork, and M. A. Pollack, "Locking of the $\mathrm{He}-\mathrm{Ne}$ laser modes induced by synchronous intracavity modulation," A ppl. Phys. Letters, vol. 5, p. 4, 1964.

[2] M. DiDomenico et al., "Generation of ultrashort optical pulses by mode locking the YAG:Nd laser," Appl. Phys. Letters, vol. 8, p. 180, 1966.

[3] A. Yariv, "Internal modulation in multimode laser oscillators," J. A ppl. Phys., vol. 36, p. 388, 1965.

[4] T. Deutsch, "Mode-locking effects in an internally modulated ruby laser," Appl. Phys. Letters, vol. 7, p. 80, 1965.

[5] A. J. DeMaria et al., "Mode locking of a $\mathrm{Nd}^{3+}$-doped glass laser," Appl. Phys. Letters, vol. 8, p. 22, 1966.

[6] R.W. Hellwarth, Advances in Quantum Electronics, J. R. Singer, Ed. New York: Columbia University Press, 1961, pp. 334-441.

[7] H. W. Mocker and R. J. Collins, "Mode competition and self-locking effects in a Q-switched ruby laser," Appl. Phys. Letters, vol. 7, p. 270, 1965.

[8] A. J. DeMaria, D. A. Stetser, and H. Heynau, "Self modelocking of lasers with saturable absorbers," A ppl. Phys. Letters, vol. 8, p. 174, 1966.

[9] J. P. Laussade and A. Yariv, "Mode locking and ultrashort laser pulses by anisotropic molecular liquids," Appl. Phys. Letters, vol. 13 , p. 65, 1968 .

[10] J. Comly, E. Garmire, J. P. Laussade, and A. Yariv, "Observation of mode locking and ultrashort optical pulses induced by anisotropic molecular liquids," Appl. Phys. Letters, vol. 13, p. 176,1968

[11] J. Frenkel, Kinetic Theory of Liquids. New York: Dover, 1955 , p. 283.

[12] P. Debye, Polar Molecules. New York: Dover, 1929.

[13] J. C. Slater, Microwave Electronics. Princeton, N. J.: Van Nostrand, 1950, p. 57 .

[14] A. Yariv, "Parametric interactions of optical modes," IEEE $J$. Quantum Electronics, vol. QE-2, pp. 30-37, February 1966.

[15] M. Paillette, "Mesures de l'effet Kerr induit par une onde lumineuse intense," Compt. Rend. Acad. Sci. (Paris), vol. 262B, pp. 264-267, January 24, 1966.

[16] See, for example, W. J. Moore, Physical Chemistry. Englewood Cliffs, N. J.: Prentice-Hall, 1950, p. 421.

[17] C. W. Cho, N. D. Foltz, D. H. Rank, and T. A. Wiggins, "Stimulated Rayleigh scattering," Phys. Rev. Letters, vol. 18, p. $107,1967$.

[18] F. De Martini, C. H. Townes, T. K. Gustafson, and P. L. Kelley, "Self steepening of light pulses," Phys. Rev., vol. 164, pp. 312323, December 1967. 